\title{
Chaos theory infects civil engineers
}

\section{Recent interest in the theory of chaos has been prompted largely by the wish to understand the inexplicable. But there may be serious practical consequences for engineers.}

GIVEN the degree to which most people's daily lives depend on engineered structures, it is a relief that civil engineers have now been infected by the preoccupation with chaos that seems for the past three years to have gripped mathematicians, physicists and even biologists. Chaotic systems are those in which the behaviour of a strictly well-determined system, mechanical or otherwise, cannot be simply inferred from a knowledge of its behaviour in a few selected circumstances (see Nature 303, 15; 1983). The simplest image is movement of a metal sphere from the top to the bottom of a pinball machine - every interaction of the sphere with an obstacle is governed by newtonian mechanics, but the outcome (the position of the ball on the bottom of the machine) is so sensitively dependent on the initial conditions that it seems to be randomly determined. If engineered structures were commonly plagued by such behaviour, we should all sleep less easily at nights.

In the most recent issue of Proceedings of the Royal Society (A307, 407-427; 1983), Dr J.M.T. Thompson of University College London points to a class of engineered structures whose behaviour may indeed be chaotic. Thompson's calculations stem from another fashion among British researchers - the design of marine structures such as those used for extracting petroleum from the North Sea. As a potential source of chaos, he offers the oscillation of an oil-production facility pinned to the ocean floor and supported more or less vertically by buoyancy. Unencumbered, such a structure is simply an easily calculable inverted pendulum, but if an oil tanker should be attached to it by mooring lines, the problem becomes more complicated. For if the top of the platform should be displaced towards the moored ship, the restoring force will simply be the buoyancy, but if the displacement is in the other direction, the force constant will be increased by the elasticity of the mooring lines.

The result is a bilinear oscillator - in each half of the range of some variable $X$ (which is a function of the time $t$ ), the motion is governed by a second order differential equation of the usual form but with different parameters. The free oscillations of such a system are easily dealt with - simply write down the general

The address of Mr Peter Stanbury (see Nature 7 July, p.11) is Flat 2, 3 Ferndale, Tunbridge Wells, Kent. analytical solutions for greater than and less than zero and match them (by velocity) in the middle. Whatever the initial conditions, the outcome is a periodic motion whose frequency is the harmonic mean of free oscillations in the two separate regimes. Damping is a complication, as a little algebra will show, while the problem of vibrations driven, for example, by wave motion in the marine environment makes the search for explicit analytical solutions impracticable.

Thompson's computer program is an allsinging all-dancing affair. The case of a ship tethered to a jetty is dealt with simply by supposing that at each impact it bounces back with the same velocity. The mechanical equations are put into a non-dimensional form, in which case the behaviour of each bilinear system can be specified by three parameters representing damping, the ratio of the two frequencies in the two halves of the free bilinear system and the forcing frequency (again in terms of the free bilinear frequency). There is, of course, a doubly infinite range of starting conditions - position and velocity - to play with. The computer program is designed to spot periodic solutions automatically, by the condition that both the position and velocity of the object are regularly repeated. In part, the outcome is what might be expected - if the forcing frequency coincides with the bilinear frequency, resonance oscillations are excited. Not surprisingly, resonance oscillations of the unsymmetrically tethered system also appear if the forcing frequency is some integral multiple of the underlying bilinear frequency, but less conspicuously. For the tethered ship, the sixth sub-harmonic can be seen. Thompson has little to say about the resonance responses that appear on the other side of the fundamental response, at forcing frequencies that are irregularly submultiples of the underlying bilinear frequency.

Where does chaos enter this hitherto unsurprising tale? The first hint of something odd is that periodic solutions with different frequencies can apparently coexist at one and the same ratio between the forcing and basic bilinear frequency. Different initial conditions give different patterns of response. For the case of the tethered ship, for example, oscillations at the fundamental frequency of the free bilinear oscillation are to be found even when the predominant response of the system is that of the fourth sub-harmonic. This much can be checked by plotting on a graph the position and velocity of the oscillating object at intervals of the fundamental period. The fundamental oscillation is represented by just one point in such a phase space, the second sub-harmonic by two and so on. Thompson shows that variation of the parameters may provoke the kind of doubling of the period of an oscillating system now widely recognized as a prelude to chaotic behaviour. And for his version of the problem of the tethered ship, Thompson finds some values of the parameters describing his system in which the motion is not stable at all, but in which it appears to hunt unsuccessfully between different sub-harmonics.

That these circumstances correspond to what is technically known as chaos follows easily enough. Thompson shows that period-doubling accumulates as his parameters are varied, eventually becoming infinite. One curious feature of the results is that within this regime, there are particular narrow ranges of the parameters in which apparently regular periodic oscillations can occur - but whether they do must depend on a particular choice of starting conditions. Another demonstration that these systems are chaotic follows from computer calculations showing that the difference between the trajectories with different initial conditions may diverge exponentially even when the initial conditions are infinitesimally close together. This is that property of chaos from which ultimately derives one of its most surprising features - the recognition that the sets of points (say the sets of values of initial conditions) leading to chaos may have fractional dimensions.

The importance of Thompson's calculations is, however, more immediate. First, it is not possible to identify all the possible motions of a mechanical system by starting with some simple periodic solution and changing it a little, perhaps by a perturbation calculation. Nor is it possible to recover all possible solutions by a judicious variation of initial conditions. The trouble, Thompson points out, is that most analyses of mechanical systems carried out with the help of computer programs are at present based on the supposition that small variations in the quantities specifying a mechanical system yield small changes in its behaviour. In at least one set of practical circumstances, Thompson shows this supposition to be incorrect.

John Maddox 